Laser Chem. 1985, Vol. 5 , pp. 167-17.2

0278-6273/85/0503-0167\$12.00/0

(C) harwood academic publishers gmbh and OPA Ltd.

Printed in Great Britain

SIMPLE METHOD FOR OBTAINING MULTIPLE-FREQUENCY

RADIATION FROM A SINGLE $\mathrm{CO}_{2}$ LASER

ALEXANDER V.EVSEEV AND ALEXANDER A.PURETZKY

Institute of Spectroscopy, USSR Academy of Sciences, 142092 Troitzk, Moscow Region, USSR

Abstract A simple method to obtain from a single $\mathrm{CO}_{2}$ laser a multiple-frequency radiation suitable for experiments in laser chemistry is suggested and experimentally realized.

In laser chemistry and laser isotope separation, there is a need for a simple and convenient multiple-frequency radiation source to conduct many processes depending on the IRmultiphoton excitation (MPE) of molecules [1]. For example, such a radiation is necessary to overcome through resonance the lower discrete vibrational states of relatively smallsized molecules, hence to accomplish their effective photolysis in moderate IR-laser fields. Suggested in this work is a simple method to achieve multiple-frequency lasing in a single $\mathrm{CO}_{2}$ laser.

In essence, the method consists in using different regions of the $\mathrm{CO}_{2}$ laser medium to effect lasing on different lines. Indeed, for the majority of stable resonators, the cross-sectional dimensions of the TEM ${ }_{\text {OO }}$ mode are comparatively small [2]. Therefore, with this mode being selected, the active medium is only partially occupied by electromagnetic 
field and so, the rest of the medium can be used to achieve lasing on other lines. In particular, such a concurrent lasing on different lines can be realized in an ordinary TEA $\mathrm{CO}_{2}$ laser whose cavity is formed by a spherical mirror with a curvature of radius $\mathrm{R}$ and a grating (Figure 1 ).

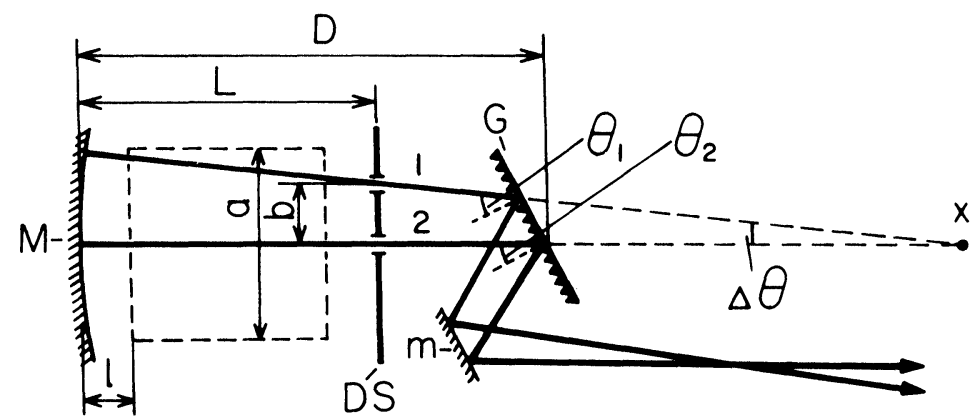

FIGURE 1. Schematic of a multiple-frequency TEA $\mathrm{CO}_{2} \mathrm{la-}$ ser. The laser cavity is formed by a spherical mirror (M) with a curvature of radius $R=5.4 \times 10^{2} \mathrm{~cm}$ and a plane grating (G) with a period of $\mathrm{d}=10^{-3} \mathrm{~cm}$. The cavity parameters were as follows. (1) Double-frequency lasing: cavity length $\mathrm{D}=1.13 \times 10^{2} \mathrm{~cm}$; distance between the diaphragm system (DS) and the mirror, $\mathrm{L}=10^{2} \mathrm{~cm}$; diaphragm aperture radius $r=0.37 \mathrm{~cm}$; distance between the centers of the aperture diaphragms, $b=1.75 \mathrm{~cm}$; cross-sectional dimension of the active medium, $a \simeq 3 \mathrm{~cm}$; distance between the mirror and the nearest boundary of the active medium, $1 \simeq 5 \mathrm{~cm}$. (2) Triple-frequency lasing: $\mathrm{L}=75 \mathrm{~cm}$; $r=0.3 \mathrm{~cm} ; \mathrm{b}=1.1 \mathrm{~cm} ; \mathrm{m}$ - tilting mirror; $\mathrm{x}$ - center of curvature of the spherical mirror.

In such a cavity, the grating in its first order operates in an autocollimation mode, i.e. for a radiation of a certain wavelength $\lambda$, the grating acts as a plane mirror set at right angles to the axis of the cavity. To extract the radiation from the cavity, use is made of the grating in its zero order. The field of the TEM ${ }_{00}$ mode in such a cavity 
is concentrated in the vicinity of the axis passing through the center of curvature of the mirror. One simple way to realize multiple-frequency lasing is to spatially divide the laser medium by a set of aperture diaphragms. Figure 1 illustrates a most simple case where two such diaphragms are used. With this method of separating the active medium, the autocollimation conditions for beams 1 and 2 are satisfied with different wavelengths $\lambda_{1}$ and $\lambda_{2}$ given by

$$
\lambda_{i}=2 d \sin \theta_{i}
$$

where $i=1$ or 2 , $d$ is the period of the grating, and $\theta_{i}$ the angle between the $i$ th beam and the normal to the grating. For the difference in wavelength between beams 1 and 2 , we have from Eq. (1)

$$
\lambda_{1}-\lambda_{2}=b\left(4 d^{2}-\lambda_{1}^{2}\right)^{1 / 2} /(R-L)
$$

where $\mathrm{b}$ is the distance between the centers of the diaphragms, $R$ the radius of curvature of the mirror, and $L$ the distance between the mirror and the diaphragm system.

The frequency of beam 1 is set in the usual way by appropriately adjusting the angle of the grating. The frequency of beam 2 can be changed relative to that of beam 1 of the following ways (see Eq. (2)): (1) by varying the distance b between the centers of the diaphragms, (2) by varying the radius of curvature of the mirror, $R$, and (3) by varying the distance $L$ between the mirror and the diaphragm system.

The simplest and most convenient way is to vary the mutual disposition of the diaphragms (i.e. the parameter $b$ ). In this case, the minimum frequency difference $\Delta \nu_{\min }$ is go- 
verned by the condition that the corresponding fields should not overlap in the cavity:

$b \geqslant 2 r$

where $r$ is the aperture radius of the diaphragms. The maximum frequency difference $\Delta \boldsymbol{\nu}_{\max }$ depends on the cross-sectional dimension of the active medium and the position of the medium in the cavity and is determined by the condition

$b \leqslant a(R-L) /(R-1)$

where $\mathrm{a}$ is the cross-sectional dimension of the active medium and 1 the distance between the mirror and the nearest boundary of the medium. For our laser (see caption to Fig. 1), $\Delta \boldsymbol{\nu}_{\min }$ and $\boldsymbol{\Delta} \boldsymbol{\nu}_{\max }$ were 2 and $14 \mathrm{~cm}^{-1}$, respectively. For example, with the frequency $\boldsymbol{\nu}_{1}$ of beam 1 being fixed on the 9R(8) line $\left(1070.5 \mathrm{~cm}^{-1}\right)$, the frequency $\boldsymbol{\nu}_{2}$ of beam 2 could be changed from $1073.3 \mathrm{~cm}^{-1}, 9 \mathrm{R}(12)$ line to $1084.6 \mathrm{~cm}^{-1}$, 9R(30) line by varying the distance between the centers of the diaphragms. The tuning curves for the fixed $\mathrm{b}=1.75 \mathrm{~cm}$ are presented in Figure 2 .

The delay time between the laser pulses of frequencies $\boldsymbol{\nu}_{1}$ and $\boldsymbol{\nu}_{2}$ could be varied over the range (0 to 200) $\pm 20 \mathrm{~ns}$ by introducing appropriate losses into one of the channels (channel 1 or 2). For our $\mathrm{CO}_{2}$ laser, the maximum energies of beams 1 and 2 were around $200 \mathrm{~mJ}$.

A triple-frequency lasing was achieved in a similar way by using a system of three aperture diaphragms. In particular, at $r=0.3 \mathrm{~cm}, \mathrm{~b}=1.1 \mathrm{~cm}$, and $\mathrm{L}=70 \mathrm{~cm}$, we obtained a triple-frequency radiation with frequencies $\boldsymbol{\nu}_{1}=1070.5 \mathrm{~cm}^{-1}$, 
SIMPLE METHOD FOR OBTAINING MULTIPLE-FREQUENCY RAD.

9R(8) line; $\boldsymbol{\nu}_{2}=1076 \mathrm{~cm}^{-1}, 9 \mathrm{R}(16)$ line; and $\boldsymbol{\nu}_{3}=1081.1$ $\mathrm{cm}^{-1}, 9 \mathrm{R}(24)$ line. The radiation energy at each of these frequencies was about 50 - $100 \mathrm{~mJ}$. By increasing the cross-sectional dimensions of the active medium one can broaden the

\section{WAVENUMBER, $\mathrm{CM}^{-1}$}

$1084.6 \quad 1078.6 \quad 1071.9$ $1055.6 \quad 1046.8 \quad 1037.4$

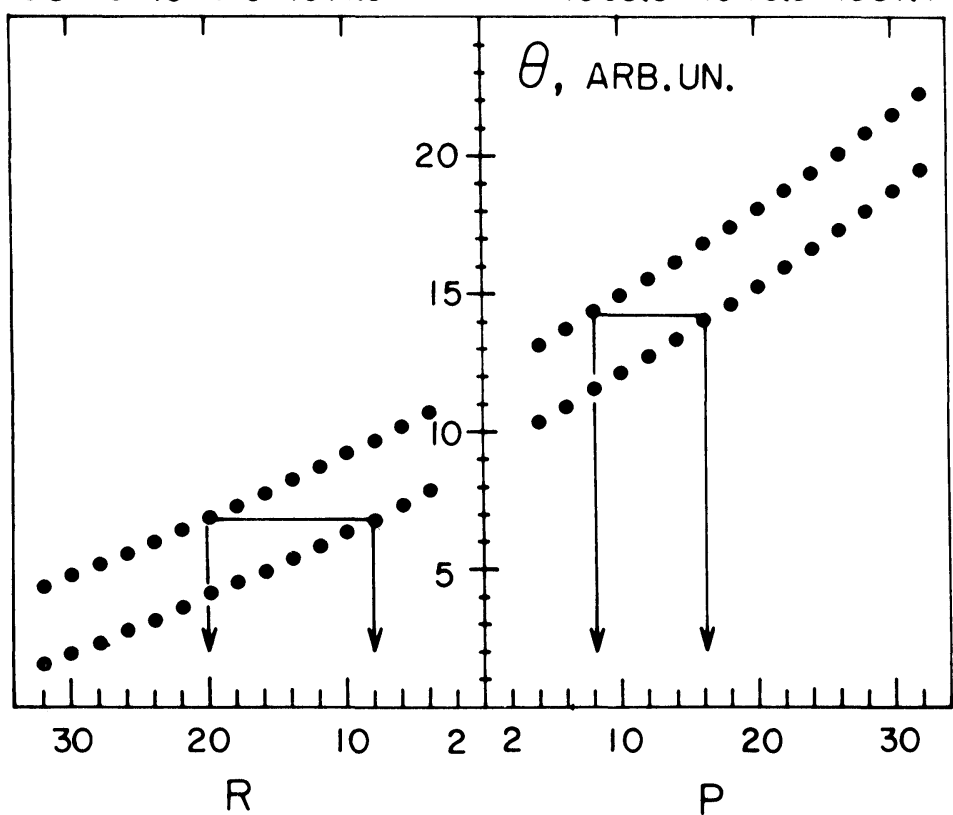

$\mathrm{CO}_{2}$ LASER LINE

Figure 2. Tuning curves for beams 1 and 2 in the case of double-frequency lasing. The cavity parameters are the same as those given in the caption to Figure 1. The arrows indicate two pairs of frequencies at which concurrent lasing is possible in this particular case.

maximum tuning range $\Delta \boldsymbol{\nu}_{\max }$ and further increase the number of possible radiation frequencies.

An important feature of the present scheme for obtaining 
multiple-frequency radiation is that the beams of different frequencies propagate at small angles to one another:

$$
\theta=\mathrm{b} /(\mathrm{R}-\mathrm{L})
$$

This substancially facilitates the use of such multiple-frequency radiation in experiments on the IR-multiphoton excitation of molecules, for it eliminates the experimental difficulties associated with the need to match the beams of different frequencies.

Thus, suggested and experimentally realized is a technique for obtaining a multiple-frequency radiation from a single $\mathrm{CO}_{2}$ laser, which is convenient to use in experiments on the MPE of molecules in multiple-frequency IR-laser fields. The advantages of the technique are the following: (1) the frequency of each beam can be easily varied over sufficiently wide limits; (2) beams of different frequencies propagate at small angles to one another, hence there is practically no need to match them; (3) multiple-frequency radiation pulses are well synchronized and delay times between them can readily be varied over the range 0 to 200 ns by introducing additional cavity losses into the appropriate channel.

\section{REFERENCES}

1. V.N.Bagratashvili, V.S.Letokhov, A.A.Makarov and E.A. Ryabov, Laser Chem., 1, 211 (1983); ㄴ, 171 (1984) and subsequent articles of this review.

2. R.L.Sheffield, S.Nazemi, and A.Javan, Appl.Phys.Lett., 29, 588 (1976). 